\title{
Optical design of the Zwicky Transient Facility: a major upgrade to the 48" Schmidt Camera at Palomar Observatory
}

Daniel J. Reiley, Richard G. Dekany, Roger M. Smith, Alexandre Delacroix, Michael Feeney, et al.

Daniel J. Reiley, Richard G. Dekany, Roger M. Smith, Alexandre Delacroix, Michael Feeney, Shawn Callahan, "Optical design of the Zwicky Transient Facility: a major upgrade to the 48" Schmidt Camera at Palomar Observatory," Proc. SPIE 10590, International Optical Design Conference 2017, $105901 \mathrm{U}$ (27 November 2017); doi: 10.1117/12.2292085

SPIE Event: International Optical Design Conference - IODC 2017, 2017, Denver, United States 


\title{
Optical design of the Zwicky Transient Facility: a major upgrade to the 48" Schmidt Camera at Palomar Observatory \\ Daniel J. Reiley ${ }^{\mathrm{a}}$, Richard G. Dekany ${ }^{\mathrm{a}}$, Roger M. Smith ${ }^{\mathrm{a}}$, Alexandre Delacroix ${ }^{\mathrm{a}}$, Michael Feeney ${ }^{\mathrm{a}}$, Shawn Callahan ${ }^{\mathrm{b}}$ \\ ${ }^{a}$ Caltech Optical Observatories, California Institute of Technology, 1200 E. California Blvd, Pasadena, CA 91125 \\ ${ }^{\mathrm{b}}$ LSST Corporation, 933 N. Cherry Ave., Tucson, AZ 85721
}

\begin{abstract}
The Zwicky Transient Facility (ZTF) will be a major upgrade to the 48" Schmidt Camera at Palomar Observatory, which was initially commissioned in 1948. Although the optical design for ZTF is a relatively small part of the project, system requirements placed special constraints on the optical design. This paper presents the optical design for ZTF as well as the system requirements that drove the optical design..
\end{abstract}

Keywords: Lens system design, Mirror system design, Telescopes

\section{INTRODUCTION}

Palomar Observatory's 48-inch Samuel Oschin Telescope, shown in Figure 1, is one of the most productive survey telescopes ever built, with a dozen completed surveys since the 1950s. Its optical design is a large, early example of a Schmidt camera.

A Schmidt camera's optical design places all optical power on a primary mirror. ${ }^{1}$ Third-order coma and astigmatism are corrected by placing the aperture stop at the mirror's center of curvature, giving the chief ray a zero angle of incidence on the primary mirror. Spherical aberration is corrected by a zero-power aspheric plate at the aperture stop. Field curvature is uncorrected, so a sharp image is obtained on a curved image surface.

a)

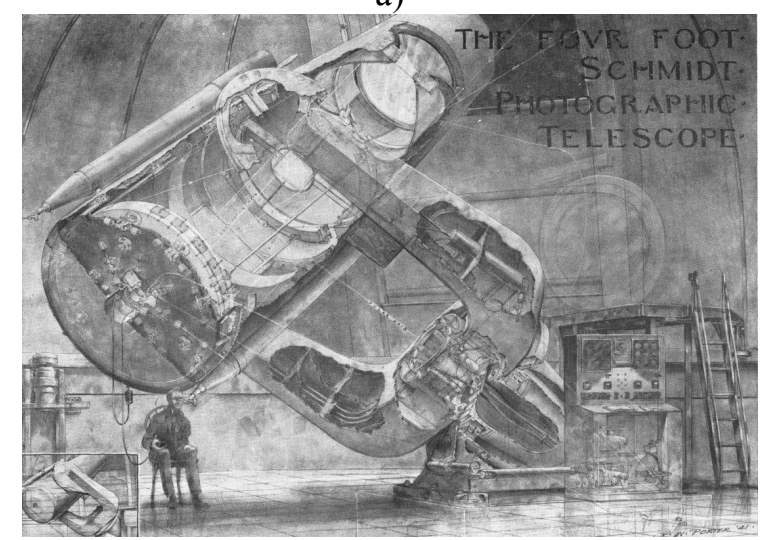

b)

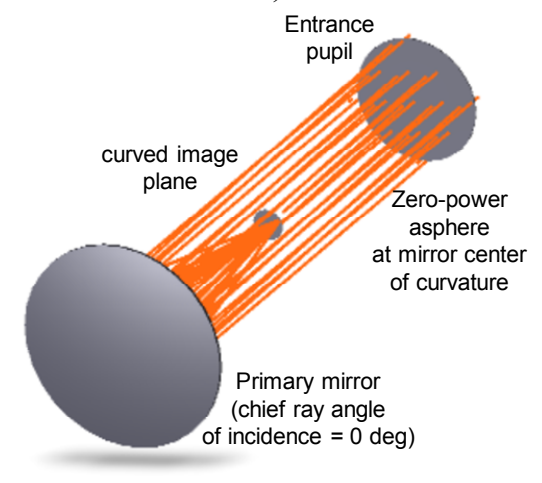

Figure 1: The original Schmidt camera at Palomar Observatory. Figure 1a shows a cutaway schematic of the full system, as originally commissioned. ${ }^{2}$ For scale, note the observer sitting near the telescope base. Figure $1 \mathrm{~b}$ shows a modern schematic of the original optical design.

The Samuel Oschin Telescope was commissioned in $1948^{3}$, achieving a $6.5 \times 6.5$ deg field of view using $355 \mathrm{~mm}$ (14") square photographic plates, which were pulled by a vacuum to a curved surface that matched the Schmidt's curved focal surface. On commissioning, the telescope achieved 3 arcsec image diameter in the $\mathrm{V}$ band. This excellent performance was key to the success of Palomar Observatory Sky Survey (POSS), which remains one of the most 
frequently used astronomical resources 60 years after its completion in 1957. Performance in these early years was limited by spherochromatism of the corrector plate and the grain size in the photographic emulsion.

The telescope has been upgraded in many important ways since the completion of this initial survey. Most importantly for this paper, in 1982 the original aspheric plate was replaced by an aspheric doublet to correct spherochromatism ${ }^{4}$, allowing the telescope to take advantage of higher-quality photographic emulsions. Since 2001, several upgrades were made to automate operations and to capture images electronically via CCDs. The advantages of CCDs also brought the disadvantage of a smaller image surface; most recently, the iPTF instrument enabled electronic imaging of a $3.5 \times 2.3 \mathrm{deg}$ field of view on the telescope. (184 x $123 \mathrm{~mm})$

The Zwicky Transient Facility expands this field of view to $7.2 \times 7.4$ deg $(376 \times 388 \mathrm{~mm}){ }^{5-9}$ somewhat larger than the original field of view. This large field of view, along with improvements to operations and data reduction pipeline, will be an important tool for transient astronomy, which includes the detection of supernovae and near-earth objects.

The large image plane is enabled by high-quality, buttable CCDs. ${ }^{10}$ ZTF uses sixteen CCD231-C6 back illuminated scientific CCD sensors from e2V, with 6144 x 6160 pixels and $15 \times 15$ um pixel size. Readout is via 4 output circuits per CCD, enabling low noise with fast readout time. The $15 \mathrm{um}$ pixel size is advantageously matched to the telescope's plate scale, producing about $1 \mathrm{arcsec} /$ pixel. This plate scale advantageously enables a toleranced, seeinglimited PSF size of about 2arcsec/pixel, which offers good balance between photometry and sensitivity.

The optical design of the telescope was modified in many important ways to achieve its wide field of view. Additional constraints were placed on the optical elements for considerations such as sensor operation, condensation, and seeing. This paper will describe the updated optical design and its rationale, show data for the optical elements, and present some on-sky data from early engineering observations. Other improvements to the telescope, unrelated to the optical design, will be left to other papers. These improvements include: a better shutter, moved to the entrance pupil to help keep the optics clean; improvements to the dome drive, which will minimize the time between pointing; new flat field hardware, which will improve image subtraction; new mounting spiders, to minimize obstruction; and a new data reduction pipeline, to minimize time to notification of important events. Relative to these improvements, the change in optical design is small in time, schedule, and budget.

a)

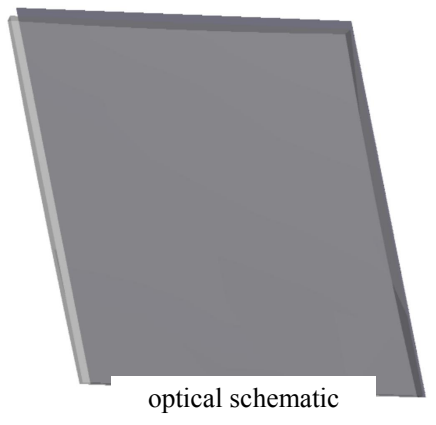

b)

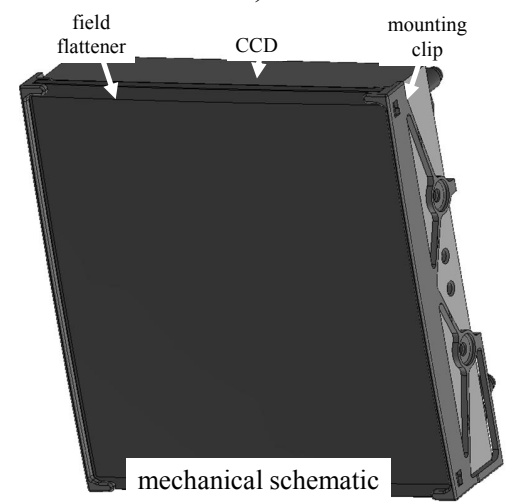

Figure 2: Optical design of the ZTF sensor/field flattener assembly (a) schematic, and b) photograph, showing the field flatteners and mounting clip.

\section{IMAGE SURFACE}

The new CCDs that enable this upgrade are flat, but the Schmidt image surface is curved. Several design elements are necessary to address this mismatch.

First, field flatteners are placed over each $\mathrm{CCD}$, as shown in Figure 2. To mount these weak positive lenses close to the CCDs and close to one another, special clips were designed. These clips, shown in Figure 2, hold the lenses in compression $2 \mathrm{~mm}$ from the CCD. Laser Metal Sintering was used to achieve the complex shape while maintaining tight tolerances. The clips were made of Titanium to match the thermal expansion of the CCD package and minimize thermal conductivity between the field flattener and the cold CCD package. To minimize stray light in the system, the 
lens edges were coated with Enthone M-series Permanent Epoxy Marking Ink. As applied, the ink outgasses and would deposit unacceptable residue on the adjacent CCDs; after a vacuum bakeout, no residue is evident.

Next, the planar CCDs are mounted as facets on a surface that nearly matches the telescope's curved image surface, as shown in Figure 3. This mounting includes the capability to shim each CCD in both piston ant tilt.

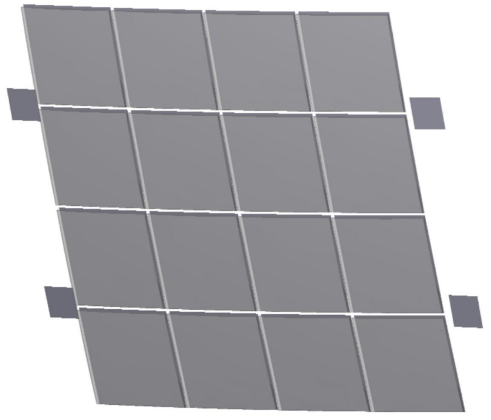

Figure 3: Optical schematic of the ZTF focal plane showing the faceted arrangement of CCDs and the field flatteners

\section{DEWAR WINDOW}

To achieve low noise readout, the CCDs must be kept at cryogenic temperatures $(<150 \mathrm{~K})$. Therefore, they must be mounted in a vacuum dewar, as shown in Figure 4. This dewar requires a window. Atmospheric pressure over the $450 \times 490 \mathrm{~nm}$ area of the dewar is about 2 tons, so safety dictated that the window be as thick as possible. Unfortunately, image quality dictated that the window be as thin as possible.

Balancing these contradictory goals required extensive modeling of the window's internal strains, as well as tight tolerances on the finish on all surfaces of the window. This tradeoff benefitted from the fact image quality is optimized with a window having a positive meniscus with the concave side in the vacuum.

a)

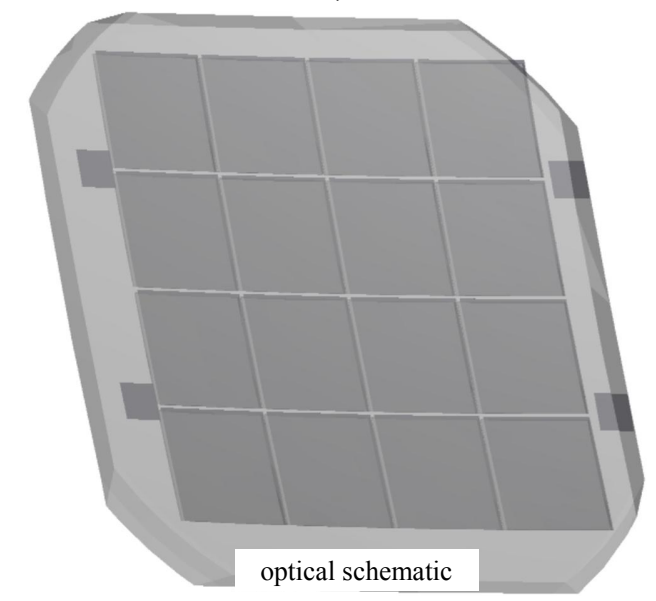

b)

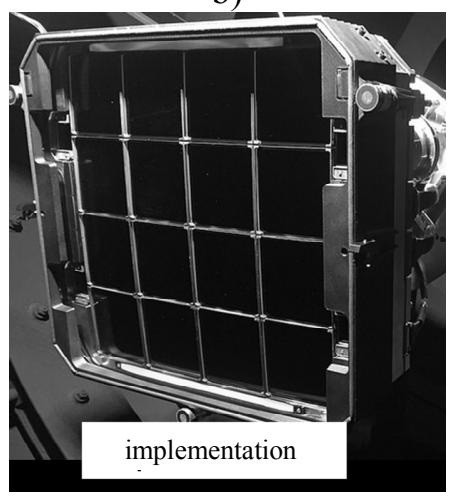

Figure 4: Optical design of the ZTF dewar assembly (a) schematic, and b) photograph, showing the window, field flatteners, and dewar walls.

The design of the window was based on the understanding that failure of amorphous materials is probabilistic. Factors affecting this probability include material choice, surface finish, and stress. Unfortunately, few of these factors were available as design variables. The only reasonable material is Fused Silica; other materials are too expensive or compromise the system's spectral bandwidth. Surface finish is constrained by the large size of the optic; we achieved a 40-20 scratch dig and further minimized the surface imperfections by polishing the edges and bevels. We were able to 
minimize stress somewhat by optimizing the o-ring design. With these design variables constrained, window thickness was the only design variable available.

Window thickness was determined by first choosing, somewhat arbitrarily, the failure probability to be $<1 \%$ in 20 years. Next the stress that would achieve this failure rate was calculated. Next, a factor of safety was chosen to be 4.8 , somewhat arbitrarily. This factor of safety means that the design stress must be 4.8 times smaller than the stress that gives the desired failure rate. With the optimized, this calculation gave a window thickness of about $32 \mathrm{~mm}$.

To prevent the cold CCDs from causing condensation on the window's outer surface, the AR coating on the window's inner surface incorporates a layer of Indium Tin Oxide (ITO). Current is then passed through this ITO layer to keep the window at ambient temperature. This ITO coating is deposited as a base layer of the coating, so additional layers must be masked to allow electrical contact to the ITO. The outer coating of the window is a nonconductive broadband AR. Both coatings pass the MIL-C-48497 standard for adhesion and severe abrasion. Both coatings achieved a max reflectivity of $0.8 \%$ and an average reflectivity of less than $0.6 \%$ in the $328-925 \mathrm{~nm}$. The maximum reflectivity on each surface occurs near a minimum in reflectivity on the other surface.

\section{BANDPASS FILTERS}

ZTF requires bandpass filters to aid classification of objects. Detailed discussions with filter ensured that the filter specifications fulfilled scientific needs while simultaneously being reasonable to fabricate; fabrication was complicated by the filters' large size $(448 \times 415 \mathrm{~mm})$. Filters in the $\mathrm{G}, \mathrm{R}$, and I bands are provided. Measured filter transmission data is shown in Figure 5. The filter is mounted immediately in front of the dewar window, and measures $450 \times 490 \times 6.2 \mathrm{~mm}$

Development of the filter transmission specifications required several iterations between the science and engineering teams. The final specification defined the transmission into four bands: a transmission band, a rejection band, and long and short transition bands. The location of the transition bands was defined at the $80 \%$ transmission points, and a minimum average transmission of $93 \%$ was required between these points. The location of the transmission bands was specified to within a tolerance of $6 \mathrm{~nm}$. The rejection band was required to have transmission $<1 \%$ outside the transmission band and the transition bands. The width of the transmission bands scaled with wavelength from $15 \mathrm{~nm}$ for the green edge of the $\mathrm{g}$ filter to $31 \mathrm{~nm}$ for the red edge of the $\mathrm{i}$ filter.

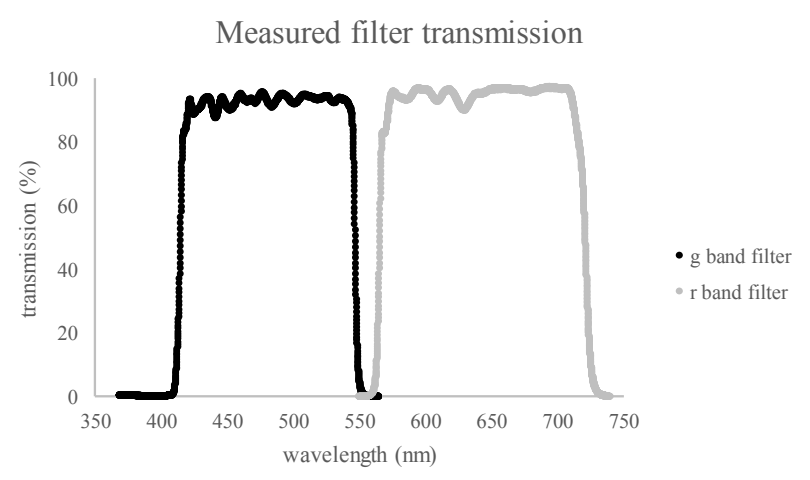

Figure 5: Measured transmission of the ZTF filters. An i band filter is in production.

\section{TRIM PLATE}

Spherical aberration is introduced into the design by the field flatteners, window, and filters. This spherical aberration is corrected by an additional aspheric plate in the entrance pupil of the telescope. This aspheric plate is $1.35 \mathrm{~m}$ diameter by $10 \mathrm{~mm}$ thick, and is figured with only a fourth-order aspheric term, with a maximum deviation of 8.0um in the clear aperture. 
a)

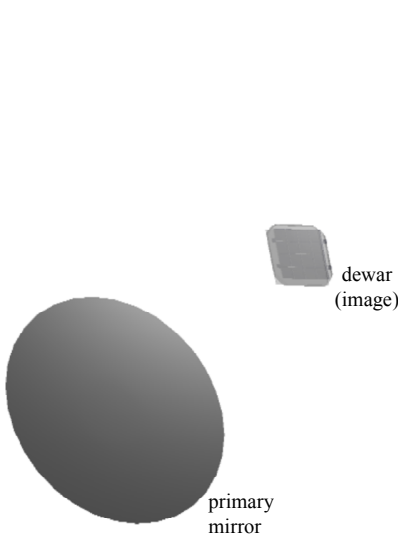

b)
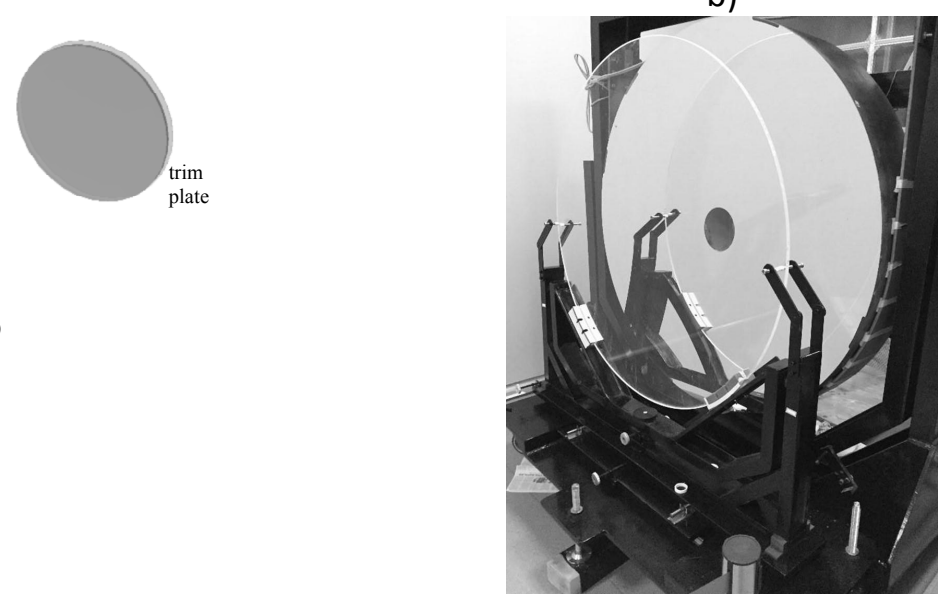

Figure 6: Schematic of the full ZTF optical system (Figure 6a) and null testing of the trim plate (Figure 6b)

\section{DATA FROM ENGINEERING RUN}

In May of 2017, the ZTF dewar was installed into the telescope as part of an engineering test. Because the trim plate was still in fabrication, image quality was compromised. However, image quality was sufficient to inform many engineering decisions.
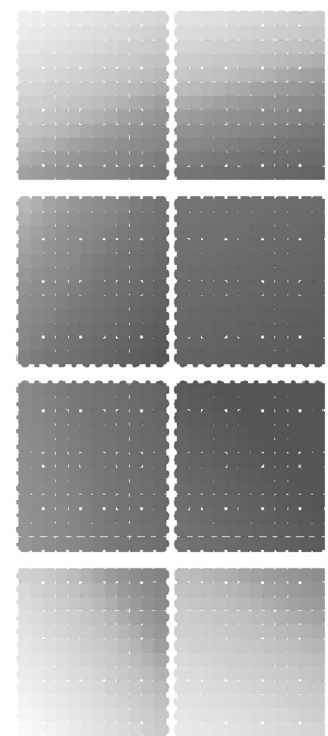
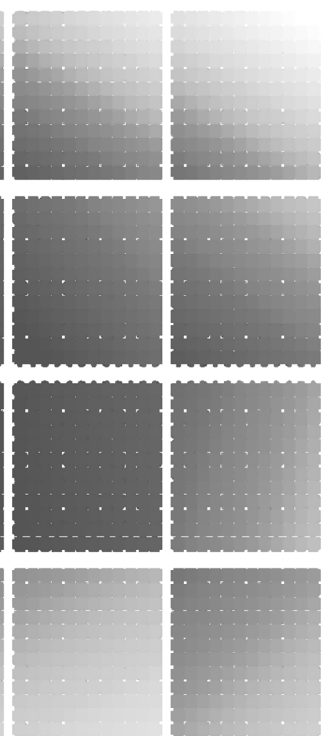

0.100

0.075

0.050

0.025

0.000

$-0.025$

$-0.050$

$-0.075$

Figure 7: On-sky measurement of the relative axial position of best focus, in mm. A general spherical error is evident, as well as tilt and focus of some CCDs

One test was to determine the proper shims to use on the CCDs. In this test, the focal plane was driven through focus while the telescope was pointed at the sky. At each focus position, the diameter of stars' images was measured, generating a plot of spot size vs. focus. Fitting these plots to a polynomial allowed the minimum to be calculated; this 
minimum represented the focus position with best image quality. Repeating this process over many stars in the field yielded focus error as a function of position on the sensors. This data, shown in Figure 7, shows a general error in curvature of the CCD surface, as well as errors in piston and tilt of individual CCDs. This data will be used to determine the proper shimming of the CCDs.

\section{FINDING TRANSIENTS}

ZTF will find astronomical transients using a data reduction pipeline that builds on the experience of the Palomar Transient Factory (PTF), the predecessor instrument on the Samuel Oschin Telescope. The PTF data reduction pipeline consists of several steps. ${ }^{11}$

The first step is image preprocessing. This step includes overscan subtraction, bias subtraction, and flat-field correction to the raw image. Since bias and flat-field features vary on long timescales, they can be updated in a separate daytime program on a cadence of a week or more. For each CCD, this program median-stacks dozens of recent bias images to make super-biases, and dozens of recent science images in $\mathrm{g}$ or $\mathrm{R}$ filters to generate super-flats in the corresponding filters. Bad-pixel masks are also updated at the same time. In ZTF, this process will be aided by the development of a new flat-field assembly

The second step is to match observations to established databases, a process called astrometric and photometric solving. Each candidate object is matched in both position and brightness to known objects in astronomy.net.

The third step is image registration. In this process, the science image and its corresponding reference image are mapped onto the same coordinate system.

The third step is image subtraction. To minimize the error on the resulting subtraction image, the PSF on the remapped reference image is converted into the PSF on the new image by a PSF convolution kernel. The convolution kernel is approximated by linear combinations of three Gaussian functions - one of which has a width narrower than the seeing, a second one has a width similar to the seeing, and the third one has a width wider than the seeing. After image subtraction, all sources with strong signal to noise are considered transient candidates. Typically, a couple hundred candidates are found on each subtracted image.

The fourth step is real/bogus classification. Many factors can produce artifacts in the subtracted image, including nonlinearity of the detectors, astrometric misalignment, imperfect convolution kernels, Poisson noise of bright objects, and cosmic rays. This classification is guided by a machine-learning algorithm that uses 42 variables, including features about the candidate shape, the quality of the subtraction, and nearby sources. The "real" training set consists of variable stars, spectroscopically classified supernovae, and asteroids, while the "bogus" set is a large number of randomly selected candidates in our database and confirmed by visual inspection, which represents the whole falsepositive population.

The final step is matching external catalogs of known astronomical transients. After removing most artifacts in the transient candidate list, the main contaminating sources are real celestial objects, including asteroids, variable stars, and active galaxy nuclei (AGNs).

\section{ACKNOWLEDGEMENTS}

ZTF is enabled by a private/public partnership, with major support from the National Science Foundation MidScale Instrumentation Program (MSIP). Private partner participation includes major commitments from California Institute of Technology(USA),Humboldt University (Germany), Lawrence Berkeley National Lab (USA), Los Alamos National Lab (USA), Oskar Klein Centre at University of Stockholm (Sweden), University of Maryland (USA), University of Washington at Seattle (USA), University of Wisconsin-Milwaukee (USA), UST Taiwan, and Weizmann Institute for Science. 


\section{REFERENCES}

[1] Smiley, C. H., “The Schmidt camera,” Popular Astronomy, Vol. 48, p.175 (1940).

[2] Harrington, R. G., "The 48-Inch Schmidt-Type Telescope at Palomar Observatory," Publications of the Astronomical Society of the Pacific, 64, 275 (1952)

[3] I. N. Reid , "The Second Palomar Sky Survey," Astronomical Society of the Pacific 103: 661-674, July 1991

[4] Smith, R. M., et. al., "The Zwicky transient facility observing system," Proc. SPIE 9147, Ground-based and Airborne Instrumentation for Astronomy (2014)

[5] Dekany, R. G., et. al., "The Zwicky Transient Facility Camera," Proc. SPIE 9908, Ground-based and Airborne Instrumentation for Astronomy VI (2016)

[6] Cao, Nugent, \& Kasliwal, "Intermediate Palomar Transient Factory: Realtime Image Subtraction Pipeline" Publications of the Astronomical Society of the Pacific, 128:114502 (2016)

[7] Kulkarni, Shrinivas R , "The Zwicky Transient Facility,“ American Astronomical Society, AAS Meeting \#227, id.314.01

[8] R.Smith, S.Kaye, "Digital CDS for the ZTF" Scientific Detector Workshop 2013, Astrophysics and Space Science Library), 2013.

[9] Eric C. Bellm and Shrinivas R. Kulkarni, "The unblinking eye on the sky," Nature Astronomy 1, Article number: $0071(2017$

[10]Eric C. Bellm , "The Zwicky Transient Facility", Proceedings, The Third Hot-wiring the Transient Universe Workshop : Santa Fe, New Mexico, November 13-15, 2013

[11] Porter, R. W., "Giants of Palomar”, California Institute of Technology (1983) 